\title{
Variable Digital Filter With Group Delay Flatness Specification or Phase Constraints
}

\author{
Hai Huyen Dam, Antonio Cantoni, Sven Nordholm, and Kok Lay Teo
}

\begin{abstract}
In this paper, we consider the design of finite-impulse response variable digital filters (VDFs) with variable cutoff frequency or variable fractional delay. We propose the design of VDFs with minimum integral squared error and constraints on the maximum error deviation in conjunction with flatness group delay specification or phase constraints. These specifications allow the VDFs to have approximately linear phase, especially in the passband. As these specifications are required to be satisfied for all the filters generated by the VDF with controllable spectral characteristics, the linear constraints resulting from the flatness specification are relaxed to inequality constraints. To make the optimization problem tractable for the phase constrained problem, suitable approximations are employed in the paper. The design problem is formulated as an optimization problem with a quadratic cost function and infinite number of constraints. A numerical scheme with adaptive grid step size is proposed for solving the optimization problem.
\end{abstract}

Index Terms-Group delay flatness, phase constraint, variable digital filter (VDF).

\section{INTRODUCTION}

$\mathbf{V}$ ARIABLE digital filters (VDFs) are digital filters with controllable spectral characteristics such as variable cutoff frequency, adjustable passband width and controllable fractional delay [1]-[3]. These spectral characteristics can be varied online. VDFs have been used in various of signal processing and communication applications. Examples include arbitrary sample rate changers, digital synchronizers and other applications involving online tuning of frequency characteristics [4]. Fractional delay digital filters have been used in timing adjustment for digital receivers [4].

In [5], the design of the VDF with least square criterion and peak gain constraints is investigated to demonstrate the trade-off between the integral squared error and the maximum error deviation. In this paper, we derive and incorporate additional constraints on the group delay flatness specification or phase constraint to allow control of the group delay or the phase in the passband.

In [6]-[9], a group delay flatness specification has been investigated for a finite-impulse response (FIR) filter. Here, we

Manuscript received March 22, 2007; revised July 26, 2007 and October 12, 2007. This paper was recommended by Associate Editor Y.-P. Lin.

H. H. Dam and K. L. Teo are with the Department of Mathematics and Statistics, Curtin University of Technology, Perth 6845, Australia (e-mail: H.Dam@@curtin.edu.au; K.L.Teo@curtin.edu.au).

A. Cantoni is with Western Australian Telecommunications Research Institute (WATRI), The University of Western Australia, Perth 6009, Australia (e-mail: cantoni@watri.org.au).

S. Nordholm is with Western Australian Telecommunications Research Institute (WATRI), Curtin University of Technology, Perth 6845, Australia (e-mail: sven@watri.org.au)

Digital Object Identifier 10.1109/TCSII.2007.912750 extend this formulation further to the VDF in which the group delay flatness specification is required to be satisfied for all VDFs generated with controllable characteristics depending on a tuning parameter. In [6] and [7], linear equality constraints are enforced to achieve the group delay flatness specification. For the VDF design problem this is not feasible since the constraints need to be satisfied for all VDFs generated by the VDF control parameter. In this paper, we employ appropriate inequality constraints since this provides sufficient freedom. The optimization problem is then formulated as a semi-infinite quadratic optimization problem with constraints involving absolute operations, in which the terms inside these operations are linear functions of the filter coefficients.

An alternative approach to include the group delay flatness specification is to control the phase of the filters generated by the VDF by incorporating additional phase constraints, especially in the passband. As such, this allows the phase of the filters generated by the VDF to have approximately linear phase or equivalent constant group delay. The design problem is formulated as an optimization problem with a quadratic cost function and nonlinear constraints. To make the design problem tractable, suitable approximations are developed in the paper. The design problem can then be approximated as a semi-infinite quadratic optimization problem. For completeness, a numerical scheme with adaptive grid step size [5], [10] is developed for solving the optimization problems resulting from the prescribed flatness specification or the phase constraints.

The paper is organized as follows. The problem formulation and optimization problem are presented in Section II. The group delay flatness specification for the VDF and the incorporation of the inequality constraints into the optimization problem are developed in Section III. A phase error approximation is outlined in Section IV. In Section V, a numerical scheme with adaptive step size is employed for solving the optimization problem. Section VI contains numerical results on a sample design of a variable delay VDF using the approaches described in this paper and also existing methods. Finally, conclusions are presented in Section VII.

\section{PRoblem Formulation AND OPtimization Problem}

Consider the design of a VDF [3] with $L$ sub-filters, depicted in Fig. 1. The frequency response of the VDF can be expressed as

$$
H\left(e^{j \omega}, \delta\right)=\sum_{l=0}^{L-1} H_{l}\left(e^{j \omega}\right) \delta^{l}
$$

where $H_{l}\left(e^{j \omega}\right), 0 \leq l \leq L-1$, is the frequency response of the $l$ th sub-filter with the corresponding impulse response $\mathbf{h}_{l}$ of length $N, \mathbf{h}_{l}=\left[h_{l}(0), \ldots, h_{l}(N-1)\right]^{T}$ and $[\cdot]^{T}$ is the transpose 


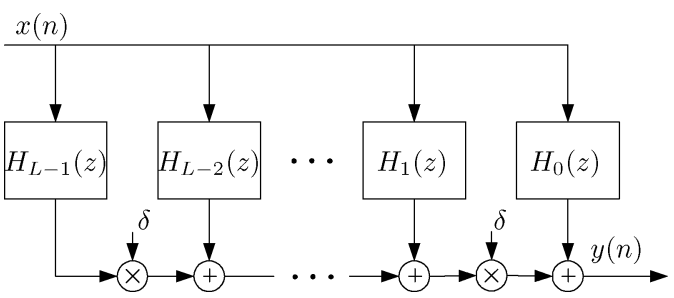

Fig. 1. VDF-Farrow structure.

operator. Also, $\delta$ is a tuning parameter varied in the range $\Delta$. The frequency response (1) can be rewritten as

$$
H\left(e^{j \omega}, \delta\right)=\sum_{l=0}^{L-1} \sum_{n=0}^{N-1} h_{l}(n) e^{-j \omega n} \delta^{l}=\mathbf{h}^{T} \mathbf{s}(\omega, \delta)
$$

where $\mathbf{h}$ and $\mathbf{s}(\omega, \delta)$ are $L N \times 1$ vectors, $\mathbf{h}=\left[\mathbf{h}_{0}^{T}, \ldots, \mathbf{h}_{L-1}^{T}\right]^{T}$ and $\mathbf{s}(\omega, \delta)=\left[\delta^{0}, \ldots, \delta^{L-1}\right]^{T} \otimes \phi\left(e^{j \omega}\right)$. The $N \times 1$ vector $\phi\left(e^{j \omega}\right)$ is given by $\phi\left(e^{j \omega}\right)=\left[1, \ldots, e^{-j \omega(N-1)}\right]^{T}$ and $\otimes$ denotes the Kronecker product.

We consider the design of a VDF with variable cutoff frequency or variable fractional delay with the desired frequency response given as

$$
H_{d}\left(e^{j \omega}, \delta\right)= \begin{cases}e^{-j \omega \tau_{d}(\delta)}, & \omega \in \mathcal{P}(\delta) \\ 0, & \omega \in \mathcal{S}(\delta)\end{cases}
$$

where $\mathcal{P}(\delta), \mathcal{S}(\delta)$, and $\tau_{d}(\delta)$ are the passband and stopband regions and the desired group delay, respectively, depending on $\delta$. By appropriate choices for $\mathcal{P}(\delta), \mathcal{S}(\delta)$ and $\tau(\delta)$, a variable cutoff frequency or variable fractional delay VDF can be specified.

The VDF is designed to minimize the integral squared error measure, defined as the integration of the weighted squared error deviation between $H\left(e^{j \omega}, \delta\right)$ and $H_{d}\left(e^{j \omega}, \delta\right)$ for all $\omega \in \Omega(\delta)$ and $\delta \in \Delta$

$$
E(\mathbf{h})=\int_{\Delta} \int_{\Omega(\delta)}\left|W(\omega, \delta)\left(H\left(e^{j \omega}, \delta\right)-H_{d}\left(e^{j \omega}, \delta\right)\right)\right|^{2} d \omega d \delta
$$

where $W(\omega, \delta)$ is a positive weighting function and $\Omega(\delta)=$ $\mathcal{P}(\delta) \cup \mathcal{S}(\delta)$. Since we consider a real coefficient vector $\mathbf{h}$, the integral squared error measure can be written as

$$
\mathbf{h}^{T} \mathbf{Q h}+\mathbf{p}^{T} \mathbf{h}+c
$$

where

$$
\begin{aligned}
\mathbf{Q} & =\mathcal{R}\left\{\int_{\Delta} \int_{\Omega(\delta)} W^{2}(\omega, \delta) \mathbf{s}(\omega, \delta) \mathbf{s}^{H}(\omega, \delta) d \omega d \delta\right\} \\
\mathbf{p} & =-2 \mathcal{R}\left\{\int_{\Delta} \int_{\Omega(\delta)} W^{2}(\omega, \delta) \mathbf{s}(\omega, \delta) H_{d}^{*}\left(e^{j \omega}, \delta\right) d \omega d \delta\right\} \\
c & =\int_{\Delta} \int_{\Omega(\delta)} W^{2}(\omega, \delta)\left|H_{d}\left(e^{j \omega}, \delta\right)\right|^{2} d \omega d \delta .
\end{aligned}
$$

$[\cdot]^{H}$ denotes the Hermitian transpose operator. Note that these double integrals can be obtained by using numerical integration [12].
In addition, the filter is designed subject to a constraint on the weighted peak deviation between $H\left(e^{j \omega}, \delta\right)$ and $H_{d}\left(e^{j \omega}, \delta\right)$,

$$
\begin{aligned}
W(\omega, \delta)\left|H\left(e^{j \omega}, \delta\right)-H_{d}\left(e^{j \omega}, \delta\right)\right| \leq & e(\omega, \delta) \\
& \forall \omega \in \Omega(\delta), \delta \in \Delta
\end{aligned}
$$

where $e(\omega, \delta)>0$ represents the allowed tolerance. In the following, we derive additional constraints for the flatness specification and the phase error approximation in the passband.

\section{GROUP Delay FlatNESS SPECIFICATION}

In this section, the group delay flatness specification for the VDF is derived. Denote by $\theta_{d}(\omega, \delta)$ the desired phase response of the VDF for a frequency $\omega$ and a tuning parameter $\delta$. From (3), we have $\theta_{d}(\omega, \delta)=-\omega \tau_{d}(\delta), \forall \omega \in \mathcal{P}(\delta)$.

Denote also by $\theta(\omega, \delta)$ the phase response of the VDF, e.g.,

$$
H(\omega, \delta)=|H(\omega, \delta)| e^{j \theta(\omega, \delta)} .
$$

The group delay response of the VDF is said to be flat to order $K$ [7], where $K$ is a positive integer, if and only if

$$
\left.\frac{d^{i} \theta(\omega, \delta)}{d \omega^{i}}\right|_{\omega=0}= \begin{cases}-\tau_{d}(\delta) & \forall \delta \in \Delta, \quad \text { if } i=1 \\ 0 & \forall \delta \in \Delta \text { and } 2 \leq i \leq K .\end{cases}
$$

As developed in [11], the condition in (5) is equivalent to the following:

$$
\left.\frac{d^{i}\left[\tan (\theta(\omega, \delta))-\tan \left(\theta_{d}(\omega, \delta)\right)\right]}{d \omega^{i}}\right|_{\substack{\omega=0 \\ \forall \delta \in \Delta \text { and } 1 \leq i \leq K .}}=0
$$

For the VDF with the frequency response in (2), the expression in (6) becomes

$$
\begin{aligned}
\tan (\theta(\omega, \delta))- & \tan \left(\theta_{d}(\omega, \delta)\right) \\
=- & \frac{\sum_{l=0}^{L-1} \sum_{n=0}^{N-1} h_{l}(n) \sin (\omega n) \delta^{l}}{\sum_{l=0}^{L-1} \sum_{n=0}^{N-1} h_{l}(n) \cos (\omega n) \delta^{l}}+\frac{\sin \left(\tau_{d}(\delta) \omega\right)}{\cos \left(\tau_{d}(\delta) \omega\right)} \\
=- & \frac{\sum_{l=0}^{L-1} \sum_{n=0}^{N-1} h_{l}(n) \sin \left(\left(n-\tau_{d}(\delta)\right) \omega\right) \delta^{l}}{\cos \left(\tau_{d}(\delta) \omega\right) \sum_{l=0}^{L-1} \sum_{n=0}^{N-1} h_{l}(n) \cos (\omega n) \delta^{l}}
\end{aligned}
$$

Given (7) and following the approach in [11], the condition (6) is satisfied if and only if:

$$
\begin{aligned}
& {\left.\left[\frac{d^{i}}{d \omega^{i}} \sum_{l=0}^{L-1} \sum_{n=0}^{N-1} h_{l}(n) \sin \left(\left(n-\tau_{d}(\delta)\right) \omega\right) \delta^{l}\right]\right|_{\omega=0} }=0 \\
& \forall \delta \in \Delta \text { and } 1 \leq i \leq K .
\end{aligned}
$$

These constraints can also be expressed as

$$
\begin{aligned}
& \sum_{l=0}^{L-1} \sum_{n=0}^{N-1} h_{l}(n)\left(n-\tau_{d}(\delta)\right)^{i} \delta^{l}=0 \\
& \forall \delta \in \Delta, i \text { is odd and } 1 \leq i \leq K .
\end{aligned}
$$


Note that (9) is only required for odd values of $i$ as (8) is always satisfied for even values of $i$. Equation (9) can be written as a linear function of the VDF coefficients $\mathbf{h}$ as

$$
\begin{aligned}
\mathbf{h}^{T} \tilde{\mathbf{s}}^{i}(\delta)= & 0 \quad \forall \delta \in \Delta \\
\text { where } \tilde{\mathbf{s}}^{i}(\delta)= & {\left[\delta^{0}, \ldots, \delta^{L-1}\right]^{T} \otimes\left[\left(0-\tau_{d}(\delta)\right)^{i},\right.} \\
& \left.\ldots,\left(N-1-\tau_{d}(\delta)\right)^{i}\right]^{T} \quad \forall \text { odd } i, 1 \leq i \leq K .
\end{aligned}
$$

The linear constraints in (10) will lead to an overly constrained problem. Thus, instead of including an infinite number of linear equality constraints in the designed problem we propose to relax these constraints by introducing inequality constraints for the flatness specification of the design. Now the design of the VDF with the integral square error as the design criterion and the constraints involving the weighted peak frequency response deviation in addition to the group delay flatness constraints can be stated as

$$
\left\{\begin{array}{l}
\min _{\mathbf{h}} \mathbf{h}^{T} \mathbf{Q h}+\mathbf{p}^{T} \mathbf{h}+c \\
W(\omega, \delta)\left|\mathbf{h}^{T} \mathbf{s}\left(e^{j \omega}, \delta\right)-H_{d}\left(e^{j \omega}, \delta\right)\right| \leq e(\omega, \delta) \\
\quad \forall \omega \in \Omega(\delta), \delta \in \Delta \\
\left|\mathbf{h}^{T} \tilde{\mathbf{s}}^{i}(\delta)\right| \leq \epsilon_{g} \quad \forall \delta \in \Delta, \text { odd } i, 1 \leq i \leq K
\end{array}\right.
$$

where $\epsilon_{g}$ is an upper bound on the group delay flatness specification. The two constraints in (11) involve absolute value operations with the terms inside the absolute operators are linear function of the filter coefficients. Thus, the problem (11) has a convex cost with convex constraints and if a feasible solution exists then the optimum solution is unique.

\section{Phase Error Approximation In the Passband}

An alternative method to including the flatness specification in Section IV is to control the phase of the filters generated by the VDF. This can be achieved by incorporating phase constraints, especially in the passband. In order words, we seek to constrain the phase response of the filters within the desired phase response with a small error $\epsilon_{p}$ in the passband

$$
\left|\theta(\omega, \delta)-\theta_{d}(\omega, \delta)\right| \leq \epsilon_{p} \quad \forall \omega \in \mathcal{P}(\delta), \delta \in \Delta .
$$

Since the phase error is a nonlinear function of the filter coefficient vector $\mathbf{h}$, the design problem incorporating additional phase constraints is highly nonlinear. These nonlinear constraints for the phase, however, can be approximated by their respective linear constraints, leading to a tractable design problem.

Since

$$
\begin{aligned}
\sin \left(\theta(\omega, \delta)-\theta_{d}(\omega, \delta)\right)=\theta(\omega, \delta)- & \theta_{d}(\omega, \delta) \\
& +O\left(\left(\theta(\omega, \delta)-\theta_{d}(\omega, \delta)\right)^{3}\right)
\end{aligned}
$$

for a small phase error $\epsilon_{p}$ in the passband, the left-hand size in (12) is approximated by the following [13]:

$$
\begin{aligned}
& \theta(\omega, \delta)-\theta_{d}(\omega, \delta) \approx \mathcal{I}\left\{\frac{H\left(e^{j \omega}, \delta\right)}{A(\omega, \delta)} e^{-j \theta_{d}(\omega, \delta)}\right\} \\
& \forall \omega \in \mathcal{P}(\delta), \delta \in \Delta
\end{aligned}
$$

where $A(\omega, \delta)$ is the filter magnitude response and $\mathcal{I}\{\cdot\}$ denotes the imaginary part of a complex number $\{\cdot\}$. By modifying the proof in [13] for the current case that includes the tuning parameter $\delta$, it can be shown that the constraint (4) results in the following constraint on $A(\omega, \delta)$ in the passband

$$
1-\frac{e(\omega, \delta)}{W(\omega, \delta)} \leq A(\omega, \delta) \leq 1+\frac{e(\omega, \delta)}{W(\omega, \delta)} \quad \forall \omega \in \mathcal{P}(\delta), \delta \in \Delta .
$$

By assuming $e(\omega, \delta) / W(\omega, \delta) \ll 1$ for all $\omega \in \mathcal{P}(\delta)$ and $\delta \in$ $\Delta$, it follows from (13) that the constraint (12) is reduced to

$$
\left|\mathcal{I}\left\{H\left(e^{j \omega}, \delta\right) e^{-j \theta_{d}(\omega, \delta)}\right\}\right| \leq \epsilon_{p} \quad \forall \omega \in \mathcal{P}(\delta), \delta \in \Delta .
$$

Thus, the approximation of the phase error constraint for each frequency $\omega \in \mathcal{P}(\delta)$ and the tuning parameter $\delta \in \Delta$ is given by

$$
\left|\mathbf{h}^{T} \mathbf{v}_{p}(\omega, \delta)\right| \leq \epsilon_{p} \quad \forall \omega \in \mathcal{P}(\delta), \delta \in \Delta
$$

where

$$
\mathbf{v}_{p}(\omega, \delta)=\mathcal{I}\left\{\mathbf{s}(\omega, \delta) e^{-j \theta_{d}(\omega, \delta)}\right\} .
$$

The VDF is designed to minimize the integral squared error measure subject to weighted peak constraints on the frequency response in conjunction with the phase error constraints in the passband

$$
\left\{\begin{array}{c}
\min _{\mathbf{h}} \mathbf{h}^{T} \mathbf{Q h}+\mathbf{p}^{T} \mathbf{h}+c \\
W(\omega, \delta)\left|\mathbf{h}^{T} \mathbf{s}\left(e^{j \omega}, \delta\right)-H_{d}\left(e^{j \omega}, \delta\right)\right| \leq e(\omega, \delta) \\
\quad \forall \omega \in \Omega(\delta), \delta \in \Delta \\
\left|\mathbf{h}^{T} \mathbf{v}_{p}(\omega, \delta)\right| \leq \epsilon_{p} \quad \forall \omega \in \mathcal{P}(\delta), \delta \in \Delta .
\end{array}\right.
$$

Similar to (11), the problem (16) has a convex cost with convex constraints and hence if a feasible solution exists then the optimum solution is unique.

\section{OPTIMIZATION APPROACH}

Since the number of constraints in (11) and (16) is large, the near-active constraint optimization method using discretization in [5], [10] can be employed for solving these problems with two infinite set of absolute linear constraints. The advantage of the method is that it concentrates on the constraints near the active points. The number of near-active constraints is, in general, significantly smaller than the number of total discretization constraints. In addition, the method employs an adaptive scheme for the discretization grid set with a sequence of grid points refined gradually.

A procedure for solving the two optimization problems formulated in this paper can be extended from [5]. As (16) has two infinite sets of infinite inequality constraints, instead of obtaining one set $\mathcal{X}$ as in step 2 in [5], a set

$$
\hat{\mathcal{X}}_{1}=\{(\omega, \delta), \delta \in \hat{\Delta}, \omega \in \hat{\Omega}(\delta)\}
$$

is obtained such that

$$
W(\omega, \delta)\left|\mathbf{h}^{T} \mathbf{s}\left(e^{j \omega}, \delta\right)-H_{d}\left(e^{j \omega}, \delta\right)\right| \geq e(\omega, \delta)-\epsilon_{1}
$$


TABLE I

InTEgral Souare ERror, MaXimum Group Delay and PhaSe ERrors For DifFERENT CASES

\begin{tabular}{|c|c|c|c|c|c|c|}
\hline Cases & Design & $\begin{array}{c}\text { Integral } \\
\text { squared error }\end{array}$ & $20 \log _{10} \delta_{p}$ & $20 \log _{10} \delta_{s}$ & $\begin{array}{c}\text { Maximum passband } \\
\text { group delay error [sample period] }\end{array}$ & $\begin{array}{c}\text { Maximum passband } \\
\text { phase error [rad] }\end{array}$ \\
\hline \multirow{2}{*}{1} & GD flatness, $K=7, \epsilon_{g}=0.01$ & $-41.1 \mathrm{~dB}$ & $-34.9 \mathrm{~dB}$ & $-39.9 \mathrm{~dB}$ & 0.0080 & 0.0015 \\
\cline { 2 - 7 } & GD flatness, $K=9, \epsilon_{g}=0.01$ & $-41.0 \mathrm{~dB}$ & $-34.8 \mathrm{~dB}$ & $-39.8 \mathrm{~dB}$ & 0.0076 & 0.0034 \\
\hline 2 & Phase constraints, $\epsilon_{p}=0.0005$ & $-41.3 \mathrm{~dB}$ & $-35.1 \mathrm{~dB}$ & $-39.9 \mathrm{~dB}$ & 0.0243 & 0.0005 \\
\cline { 2 - 7 } & Phase constraints, $\epsilon_{p}=0.0002$ & $-41.3 \mathrm{~dB}$ & $-35.1 \mathrm{~dB}$ & $-39.9 \mathrm{~dB}$ & 0.097 & 0.0002 \\
\hline 3 & Least square solution & $-42.8 \mathrm{~dB}$ & $-30.3 \mathrm{~dB}$ & $-32.6 \mathrm{~dB}$ & 0.0524 & 0.0010 \\
\hline 4 & With peak error constraints & $-41.4 \mathrm{~dB}$ & $-34.9 \mathrm{~dB}$ & $-39.9 \mathrm{~dB}$ & 0.0301 & 0.0032 \\
\hline
\end{tabular}

and

$$
\hat{\mathcal{X}}_{2}=\{(\omega, \delta), \delta \in \hat{\Delta}, \omega \in \hat{\mathcal{P}}(\delta)\}
$$

is obtained such that

$$
\left|\mathbf{h}^{T} \mathbf{v}_{p}(\omega, \delta)\right| \geq \epsilon_{p}-\epsilon_{1} .
$$

As for step 3 in [5], the discretization problem of (16) is solved with $\mathcal{X}_{2}$ and $\mathcal{X}_{2}$ being the region for the first and the second constraints, respectively.

\section{DESIGN EXAMPLES}

In this section, the design procedures developed in this paper are applied to the design of a variable delay VDF. The design of other VDFs such as variable cutoff frequency can be obtained similarly. For the purpose of comparison we also present results based on the alternative design techniques proposed in [1], [3] and [5]. Thus, the following cases are considered.

- Consider the design of a VDF with peak error constraints and additional group delay flatness constraints as proposed in this paper.

- Consider the design of a VDF with peak error constraints and additional phase error approximation constraints. The upper bound on the phase error $\epsilon_{p}$ is chosen as $\epsilon_{p}=0.0005$ and $\epsilon_{p}=0.0002$, respectively.

- Finally, for the purpose of comparison with existing design techniques for VDFs we consider the design of a VDF based on:

- minimize integral squared error and no constraints as proposed in [1], [3];

- minimize integral squared error with peak error constraints as proposed in [5].

The length of the variable delay VDF designed filter to be designed is $N=21$ with $L=5$. The range of $\delta$ is chosen as $\Delta=$ $[0,1]$. The desired delay changes linearly over one sample delay with $\tau_{d}(\delta)=\tau_{d}+\delta$ with the desired delay $\tau_{d}=10$. The passband and stopband regions are given as $\mathcal{P}(\delta)=[-0.2 \pi, 0.2 \pi]$ and $\mathcal{S}(\delta)=[-0.5 \pi,-0.4 \pi] \cup[0.4 \pi, 0.5 \pi]$. The weighting function $W(\omega, \delta)$ is one for all frequencies. The passband and stopband ripple measures in $\mathrm{dB}, 20 \log _{10} \delta_{p}$ and $20 \log _{10} \delta_{s}$, are required to be less than $-35 \mathrm{~dB}$ and $-40 \mathrm{~dB}$, respectively, where with $\delta_{p}$ and $\delta_{s}$ being the passband and stopband ripples. The number of discretized points for $\delta$ is 128 while the number of discretized points for $\omega$ is 256 .

Table I shows the integral squared error, the maximum passband, stopband errors and the maximum phase, group delay errors in the passband for the four cases considered.
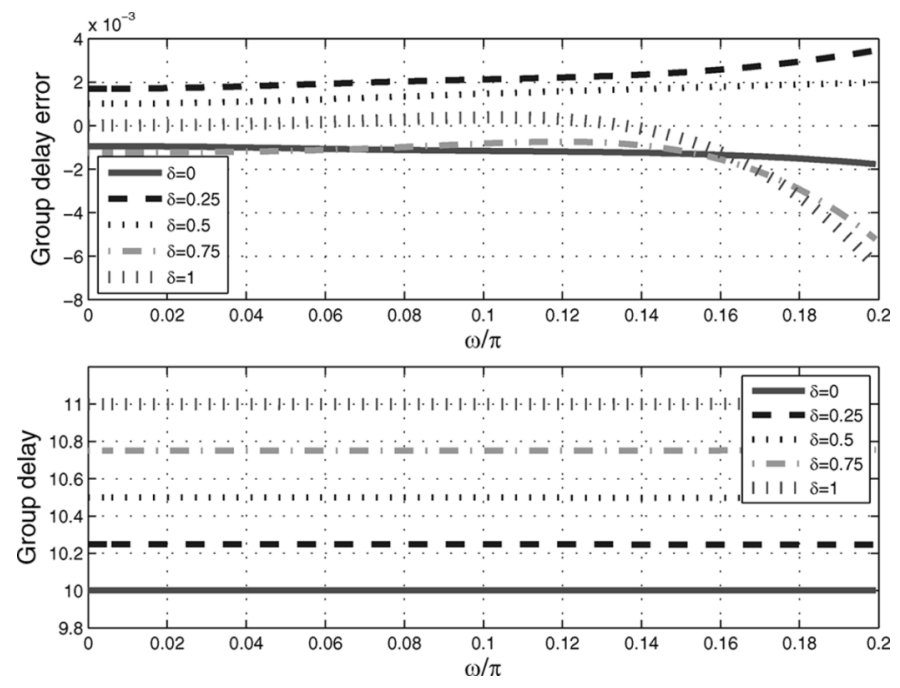

Fig. 2. Group delay response and group delay error for the group delay flatness constraint case with $K=7$ and $\epsilon_{g}=0.01$.
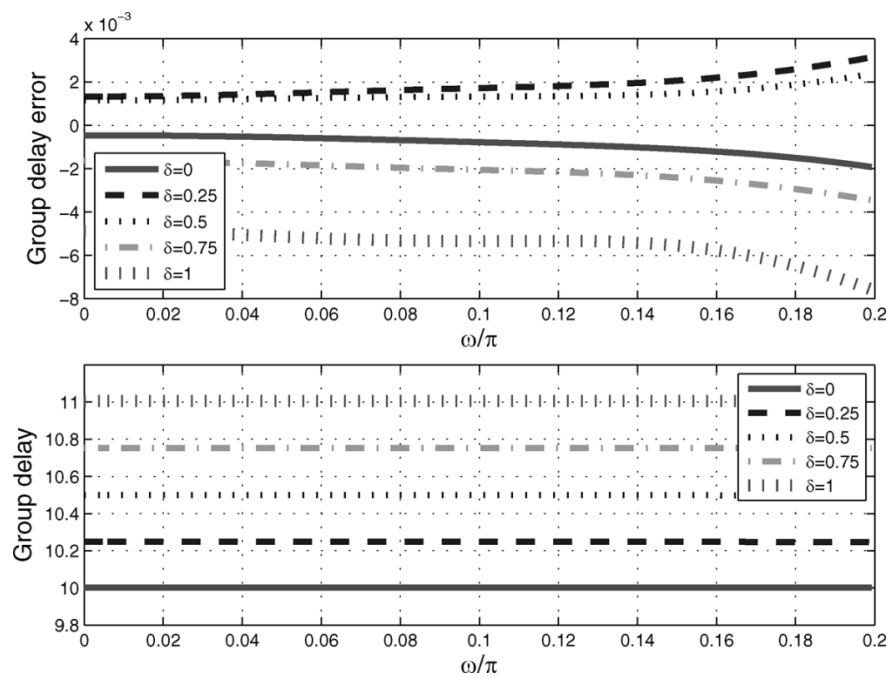

Fig. 3. Group delay response and group delay error for the group delay flatness constraint case with $K=9$ and $\epsilon_{g}=0.01$.

Figs. 2 and 3 show the group delay error and the group delay of the VDFs with group delay flatness specification order of $K=7$ and $K=9$, respectively, and the upper bound $\epsilon_{g}=$ 0.01 . The group delay is flat at around the frequency $\omega=0$ for all $\delta$.

Figs. 4 and 5 show the magnitude response and the phase error for the two cases with phase constraints. The phase error approximations are highly accurate with the phase error for all filters less than $\epsilon_{p}$ in the passband. Again, the phase and the group delay errors are reduced when $\epsilon_{p}$ is reduced. Also, the 

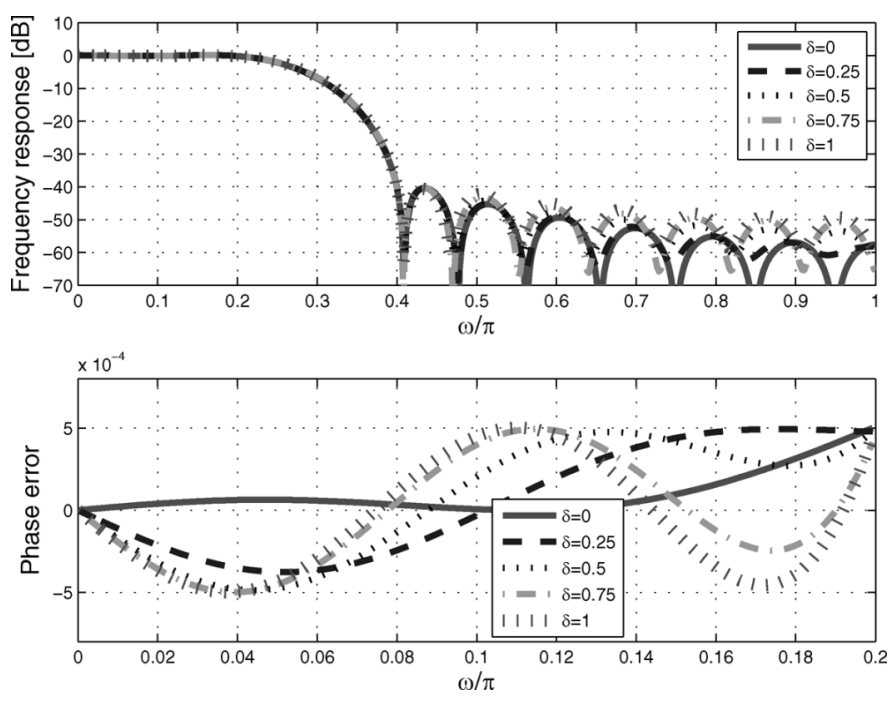

Fig. 4. Magnitude response and phase error for the phase constraint case with $\epsilon_{p}=0.0005$.
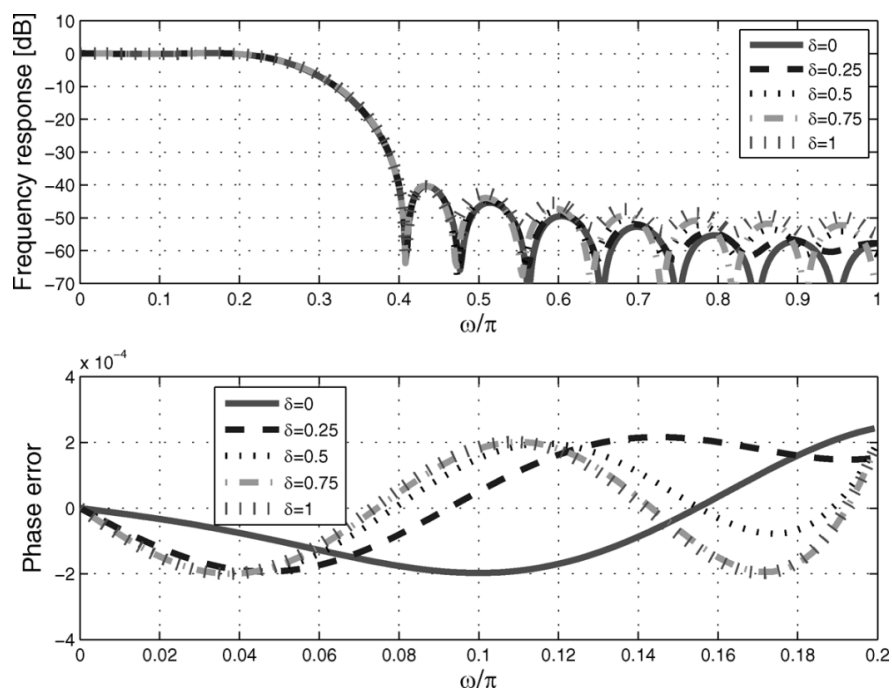

Fig. 5. Magnitude response and phase error for the phase constraint case with $\epsilon_{p}=0.0002$.

phase errors are lower than those in case 1. The group delays, however, do not have the flatness property as for those in case 1.

As expected, the integral squared error is lowest for case of unconstrained minimization of the integral squared error as proposed in [1], [3]. However, for case of unconstrained minimization of the integral squared error, the passband ripple, stopband ripple and maximum passband group delay error are significantly higher than for the design approach proposed in this paper.

From Table I it is also clear that the design based on minimum integral squared error with peak error constraints as proposed in
[5] yields slightly lower integral squared error when compare to the designs based on this paper. The maximum passband group delay error, however, is higher than those obtained using the design methods based on this paper.

\section{CONCLUSION}

In this paper, we incorporate the flatness group delay specification or the phase constraint into the design of a VDF with minimum integral squared error measure in conjunction with weighted peak constraint deviation on the frequency response. These additional specifications allow the control on the group delay or the phase of the filters generated by the VDF with controllable spectral characteristics, especially in the passband. Design examples show the trade-off between the phase, the group delay errors and the integral squared error. In addition, comparable performance is obtained by incorporating the flatness group delay specification or the phase constraints.

\section{REFERENCES}

[1] C. K. S. Pun, S. C. Chan, K. S. Yeung, and K. L. Ho, "On the design and implementation of FIR and IIR digital filters with variable frequency charactericstics," IEEE Trans. Circuits Syst. II, Analog Digit. Signal Process., vol. 49, no. 11, pp. 689-703, Nov. 2002.

[2] H. Johansson and P. Lowenborg, "On the design of adjustable fractional delay FIR filters," IEEE Trans. Circuits Syst. II, Analog Digit. Signal Process., vol. 50, no. 4, pp. 164-169, Apr. 2003.

[3] C. W. Farrow, "A continuously variable digital delay element," in Proc. IEEE Int. Symp. Circuits Syst., Jun. 1988, vol. 3, pp. 2641-2645.

[4] T. I. Laakso, V. Valimaki, M. Karjalainen, and U. K. Laine, "Splitting the unit delay: Tools for fractional delay filter design," IEEE Signal Process. Mag., vol. 13, no. 1, pp. 30-60, Jan. 1996.

[5] H. H. Dam, A. Cantoni, K. L. Teo, and S. Nordholm, "Variable digital filter with least square criterion and peak gain constraints," IEEE Trans. Circuits Systems II, Exp. Briefs, vol. 54, no. 1, pp. 24-28, Jan. 2007.

[6] K. M. Tsui, S. C. Chang, and K. S. Yeung, "Design of FIR digital filters with prescribed flatness and peak error constraints using second-order cone programming," IEEE Trans. Circuits Systems II, Exp. Briefs, vol. 52, no. 9, pp. 601-605, Sep. 2005.

[7] R. A. Gopinath, "Least squared error FIR filters with flat amplitudes or group delay constraints," IEEE Signal Process. Lett., vol. 10, no. 9, pp. 270-272, Sep. 2003.

[8] I. W. Selesnick and C. S. Burrus, "Maximally flat low-pass FIR filters with reduced delay," IEEE Trans. Circuits Systems II, Analog Digit. Signal Process., vol. 45, no. 1, pp. 53-68, Jan. 1998.

[9] S. Samadi, A. Nishihara, and H. Iwakura, "Universal maximally flat lowpass FIR systems," IEEE Trans. Signal Process., vol. 48, no. 7, pp. 1956-1964, Jul. 2000.

[10] K. L. Teo, X. Q. Yang, and L. S. Jennings, "Computational discretization algorithms for functional inequality constrained optimization," Ann. Oper. Res., pp. 215-234, 2000.

[11] J. P. Thiran, "Recursive digital filters with maximally flat group delay," IEEE Trans. Circuit Theory, vol. CT-18, pp. 659-664, Nov. 1971.

[12] R. L. Burden and J. D. Faires, Numerical Analysis. Stamford, CT: Thomson Higher Education, 2005.

[13] H. H. Dam, A. Cantoni, K. L. Teo, and S. Nordholm, "Digital laguerre filter design with minimum passband to stopband energy ratio subject to peak and group delay constraints," IEEE Trans. Circuits Syst. I, Reg. Papers, vol. 53, no. 5, pp. 1108-1118, May 2006. 